\section{Abordaje de las dificultades de comunicación en personas con discapacidad intelectual: la importancia del contexto}

\author{
An approach to communication difficulties \\ in people with intellectual disabilities: the \\ importance of context
}

Ismael Garzón Sánchez <ismaelgarzon@gorabide.com> Gorabide. España

\section{Mirko Gerolin Pelucci}

<mirkogerolin@gorabide.com>

Gorabide. España

\section{María Jesús Herrero \\ Brandin \\ <mjesusherrero@gorabide.com> \\ Gorabide. España}

Monika Mena Castañares $<$ mmena@fundacionede.org>

Fundación EDE. España

\section{Ainara Canto Combarro <acanto@fundacionede.org> \\ Fundación EDE. España}

Irama Vidorreta Gutiérrez <ividorreta@fundacionede.org>

Fundación EDE. España

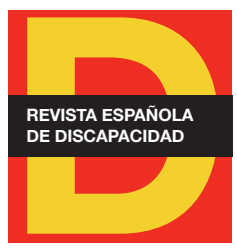

\begin{abstract}
Communication is an essential basic right for the quality of life of every person, where all the agents involved have responsibility for guarantying an effective dialogue. People with intellectual and developmental disabilities with specific communication needs, manage communicative forms that could often seem limited and difficult to comprehend for most people, therefore reducing their effectiveness. This paper proposes a procedure based on the use of alternative and augmentative communication systems with a person-centered planning methodology, where technology is put at the service of the person's life project, promoting the involvement of their relationships towards the goal of improving their quality of life, and generalizing the use of communication systems in all contexts.
\end{abstract}

\section{Abstract}

\section{Keywords}

Communication, intellectual and developmental disabilities, alternative and augmentative communication systems, person-centered planning, quality of life.

\section{Para citar:} de las dificultades de comunicación en personas con discapacidad intelectual: la importancia del contexto". Revista Española de Discapacidad, 8(I), pp. 103-127.

Doi: <https://doi.org/10.5569/23405104.08.01.06>

Fecha de recepción: 08-04-2019 Fecha de aceptación: 06-05-2020
Garzón, l. et al. (2020). “Abordaje 


\section{Introducción}

Un importante número de personas con discapacidad presentan dificultades del habla y lenguaje. Investigaciones realizadas en EEUU, Canadá, Australia y Reino Unido muestran una prevalencia que va de un 1,3\% a un 1,5\% de personas con necesidades comunicativas especiales (Beukelman y Mirenda, 2013). Basándose en la encuesta EDAD (Encuesta de Discapacidad, Autonomía Personal y situaciones de Dependencia) del año 2008, existe una prevalencia del 30, $4 \%$ en varones y 16,5 en mujeres, en edades comprendidas de 6 a 15 años. Esta aumenta hasta el 32,3\% en varones y en 22,3\% en mujeres, en edades de 55 a 64 años.

Según Calleja (2018), el perfil de la persona usuaria con Necesidades Complejas de Comunicación (NCC) se mantiene. Sin embargo, en los últimos años, se han observado cambios de índole demográfico, social y tecnológico que incrementan el número de personas usuarias que requieren una Comunicación Alternativa y Aumentativa (CAA). La mayoría de estas personas, si no todas ellas, manifiestan una intención comunicativa; esto es, muestran interés en la interacción social, haciendo buen uso de sus aptitudes no verbales como el contacto visual y la sonrisa, y emplean gestos para hacerse entender cuando no lo consiguen con palabras (Soro-Camats, 1998). Para lograr esto, es necesario un esfuerzo intencional que asegure a las personas con dificultades de comunicación tener oportunidades de interacción a lo largo del día (Chung et al., 2012; Light y McNaughton, 2014). Sin embargo, la ausencia de guías metodológicas dificulta la intervención en este ámbito (Goldbart, et al., 2014; Light y McNaughton, 2015).

Existen diferentes sistemas de comunicación, sin ayuda y con ayuda. La comunicación sin ayuda se caracteriza por emplear el propio cuerpo; aquí, tanto la motricidad fina como la motricidad gruesa, las expresiones faciales, el movimiento ocular y las posturas corporales, van configurando el mensaje (Calleja y Rodríguez, 2018). Asimismo, las vocalizaciones en personas que no han desarrollado el lenguaje oral pueden ir configurando un código de comunicación que sustituya el habla. Así, ciertos sonidos acaban convirtiéndose en un vocabulario propio inventado por la persona para comunicarse por ejemplo decir "titi" para referirse a "coche" (Calculator 2014).

De igual manera, la utilización de signos (como por ejemplo el lenguaje de signos utilizado por personas sordas), "pueden tener un valor lingüístico - cuando la configuración manual proporciona información clara de la relación entre el signo - concepto" (Calleja y Rodríguez, 2018: 166), convirtiéndose también en un recurso comunicativo para personas con necesidades especiales de comunicación que pueden oír, pero que tienen dificultades a la hora de verbalizar.

Según Calculator (2014), es posible encontrarse con sistemas de comunicación propios de cada persona que, con el paso del tiempo, su red natural aprende a identificar y a utilizar. Los gestos, expresiones faciales y sonidos que habitualmente configuran este sistema de comunicación creado por la propia persona pueden resultar limitados y difíciles de intuir dado que, al no ser un sistema estándar, no es compartido por la mayor parte de las personas, lo que, por ende, disminuye su eficacia. En consecuencia, se deben desarrollar sistemas con ayuda, que permitan un flujo de información más rico y complejo. Esta modalidad de sistemas engloba dispositivos de bajo y alto nivel tecnológico (Reichle et al., 2016), de los cuales se hablarán más adelante. 


\section{Un breve repaso a los sistemas de comunicación}

Los sistemas de comunicación aumentativos tienen como objetivo complementar el habla residual (Calleja y Rodríguez, 2018) compensando así impedimentos temporales o permanentes (American Speech-Language-Hearing Association, 2005). Los sistemas alternativos de comunicación sustituyen el lenguaje oral con herramientas, estrategias, símbolos, imágenes y dispositivos generadores de voz, que un individuo usa para resolver sus retos de comunicación diarios cuando éste no es comprensible o está ausente (International Society for Augmentative and Alternative Communication, 2017).

Según Barbosa et al. (2018), cuando se habla de apoyos de "baja tecnología" se abarcan libros de comunicación o pizarras (sin alimentación), palabras escritas en papel, fotografías, dibujos lineales y pictogramas. Al contrario, los apoyos de "alta tecnología" proporcionan acceso a computadoras personales, laptops y tablets.

Es importante resaltar que alta y baja tecnología son igualmente válidas siempre y cuando se ajusten a las posibilidades que cada persona tiene de adquirirla y usarla (Barbosa et al., 2018). Pese a ello, la experiencia con dicha población ha permitido valorar las grandes ventajas que proporcionan los apoyos de "alta tecnología" en términos de interactividad, facilidad para su transporte y a la normalidad de su uso por parte de gran parte de la ciudadanía. De acuerdo con Rochelle (2016), las nuevas tecnologías y sus aplicaciones son el avance que permitirá dar mayor asistencia a estudiantes con discapacidad en la comunicación.

Los sistemas aumentativos y alternativos de comunicación (en adelante SAAC) avanzan rápidamente gracias al aporte de las propias tecnologías de la información y de la comunicación (TIC). Éstas ofrecen sistemas cada vez más accesibles como por ejemplo el control ocular (Simacek et al., 2017). Sin embargo, es importante que, a la hora de definir un plan de comunicación, los profesionales y familias sean capaces de identificar, de entre las múltiples alternativas tecnológicas existentes, aquellas que mejor se adaptan a la realidad de la persona. De no hacerlo se corre el riesgo de, cegados por la tecnología, definir planes de comunicación descontextualizados y poco centrados en la persona usuaria (Batorowicz et al., 2018).

Hablar de sistemas alternativos y aumentativos de comunicación con o sin apoyos, de alta y baja tecnología, no significa que, a la hora de diseñar apoyos para la comunicación, cada una de estas categorías sea excluyente con respecto a las otras (Barbosa et al., 2018). De hecho, esto no es así en la práctica. Como lo refiere Soro-Camats (1998), lo habitual será que la persona con discapacidad trate de comunicarse en primera instancia utilizando su lenguaje, gestos, expresiones faciales, y complemente todo ello con sus sistemas alternativos para expresar aquello que no logra descifrarse o que requiere una mayor complejidad por parte del receptor. Lo anterior se evidencia en la investigación de Soto y Clarke (2017), en la que la combinación de SAAC dentro de una intervención basada en la comunicación genera efectos positivos en niños con trastornos graves del habla motora y retraso del lenguaje expresivo.

En la utilización de todas estas habilidades y herramientas de forma integrada, se produce lo que se denomina comunicación total, siendo importante que el interlocutor entienda la complejidad de esta e identifique las estrategias y comportamientos que facilitarán el intercambio comunicativo de la forma más acertada posible, pues los apoyos no son el fin, sino el medio (Acuña-Partal et al., 2018). Por consiguiente, es rele- 
vante decirle a la persona honestamente cuando se comprende su mensaje y cuando se necesita el uso de un sistema de comunicación alternativo. Esta misma actitud pondrá de manifiesto el interés por la persona y por lo que quiere decir, y no tanto por el soporte utilizado para expresarlo (Griffiths y Smith, 2016).

Light y colaboradores (Light, 1989; Light et al., 1998, Light, et al., 2003;) describen en detalle las competencias necesarias por parte de quienes requieren SAAC y las clasifican en cuatro grupos: competencias lingüísticas, competencias operativas, competencias sociales y competencias estratégicas. La explicación en detalle de cada una de ellas se abordará en el apartado "descripción del proceso, pasos a seguir", donde se aporta la valoración competencial de la persona destinataria del proyecto de comunicación eficaz.

Ahora bien, para garantizar una comunicación eficaz, la utilización SAAC debe enfocarse no en la demostración de habilidades aisladas dentro de laboratorios, salas de clínicas o sesiones de terapia, sino más bien en el desempeño real de la comunicación dentro de contextos naturales (González et al., 2014). Estudios como el realizado por Creer et al. (2016) reflejan la necesidad de que los SAAC sean dados en contextos naturales, pues existe cada vez más un aumento poblacional de personas que pueden ser beneficiarias potenciales de estas tecnologías de comunicación activas, como en el caso de Reino Unido.

La Clasificación Internacional del Funcionamiento, de la Discapacidad y de la Salud, de la Organización Mundial de la Salud, reconoce la importancia de centrarse en la comunicación funcional y la participación en la sociedad (Enderby, 2013; Simeonsson et al., 2012). Es por ello que al no darse estas condiciones, la generalización de lo aprendido al contexto cotidiano de la persona puede verse mermada como consecuencia de no haber tenido en cuenta los tiempos que la persona con necesidades comunicativas especiales requiere para elaborar o procesar el mensaje (la comunicación con alguien que utiliza un SAAC suele ser mucho más lenta), o el desconocimiento del SAAC por parte del entorno (esto es particularmente relevante cuando en ese entorno existen otras personas con discapacidad intelectual) (McNaughton et al., 2019).

Otro factor que incide en la accesibilidad es la usabilidad del SAAC fuera del contexto de aprendizaje. Por ejemplo, un SAAC muy voluminoso, incómodo de llevar o lento limita su uso en un entorno natural. Estos factores pueden producir ciertos efectos colaterales que tienen que ver con la dificultad a la hora de generalizar los conocimientos adquiridos a entornos vitales habituales, tal y como lo refiere Soro-Camats (1998) "la gran mayoría de los niños y adultos que necesitan usar sistemas aumentativos y alternativos de comunicación tienen también necesidades en otros aspectos de sus vidas, como puede ser (...) el entorno social, el desplazamiento y la manipulación del sistema" (pp. 63).

De esta manera, se cree relevante que al trabajar con SAAC, se tenga en cuenta dos objetivos. El primer de ellos que la comunicación es un derecho básico de todas las personas dado que es el derecho humano por el cual todos los demás derechos se hacen posibles (TASH, 2016). La competencia comunicativa es por tanto esencial para la calidad de vida de toda persona ya que proporciona los medios para desarrollar objetivos vitales en ámbitos tan diversos como la esfera personal, laboral, social entre otras muchas otras (Calculator, 2009; Lund y Light., 2007).

Segundo, además de ser un derecho, la comunicación se vertebra como un proceso en el que el significado se construye entre todas las personas que intervienen Soro-Camats (1998). Este enfoque se contrapone al tradicional, en el que el acento en la educación y el aprendizaje del sistema de comunicación recae en la 
persona con discapacidad; quedando implícito en este tipo de intervención que es ésta quien, al tener dificultades a la hora de producir o procesar el mensaje, ha de aprender a hacerlo mejor. Es necesario partir de la hipótesis de que tanto las personas con necesidades comunicativas especiales como sus interlocutores, frecuentemente, requieren de apoyos específicos para el desarrollo de una comunicación funcional (Abbeduto et al., 2001; Reichle et al., 2016; Snell et al., 2006).

Si bien adquirir competencias en el manejo de sistemas de comunicación alternativa y aumentativa es vital para mejorar la autodeterminación y la autonomía (Calculator, 2014), una aproximación más sistémica muestra que todos los agentes involucrados en un proceso comunicativo tienen su responsabilidad a la hora de garantizar un diálogo eficaz (Griffiths et al., 2019).

Finalmente, se ha de tener en cuenta que un SAAC es en esencia una ayuda técnica y por tanto ha de ser propiedad de la persona ya que ha de poder acceder a ella siempre que lo desee tal y como lo refiere Griffiths et al., (2019: 196) "el sistema debe estar disponible para la persona usuaria cuando se requiere, siendo fácilmente portátil o mediante su montaje en una silla, asegurando que el vocabulario relevante está disponible es rápido y fácilmente de seleccionar dentro del conjunto".

A partir de la experiencia de trabajo con estudiantes con necesidades de comunicación complejas, se recomienda que los equipos educativos consideren la implementación de estrategias de autogestión y responsabilicen a estudiantes de llevar sus propios dispositivos (Chung y Douglas, 2014). Es tan paradójico utilizar un SAAC solamente en el aula como lo sería que una persona que necesita una silla de ruedas para desplazarse tuviera acceso a ella solo cuando está en casa. Otro escenario que normalmente se genera, es que no hay un interés compartido sobre el que sustentar el proceso comunicativo entre emisor y receptor (Pérez y De lima, 2017). En estos casos, la intención comunicativa se desvanece igual que lo hace cualquier comportamiento que no sirve para lograr nuestros objetivos. Esto se refleja en la investigación realizada por Acuña-Partal et al. (2018), donde se demuestra que las personas con discapacidad intelectual al sentirse incomprendidas, no recibir una latencia suficiente para dar una respuesta y reconocer poco interés de la otra persona, abandonan la conversación o no la inician.

Estudios en niñas y niños con dificultades de comunicación demostraron que los eventos de comunicación ocurren principalmente con adultos y se observaron muy pocas iniciaciones espontáneas. En casi la mitad de estas oportunidades, los estudiantes no tuvieron acceso a su sistema de comunicación. Los resultados indicaron que se necesitan esfuerzos más deliberados para garantizar que las personas tengan el acceso adecuado y la oportunidad de utilizar los sistemas SAAC en todos los entornos (Andzik et al., 2016).

Dada las escasas evidencias, y al reto y oportunidad que supone una tecnología que evoluciona rápidamente, se necesita potenciar la investigación en comunicación efectiva y eficiente para personas con discapacidades severas, profundas y múltiples (Simacek, et al., 2017). 


\section{Por qué vincular la planificación centrada en la persona (PCP) a un sistema de comunicación alternativo}

La PCP nace a mediados de los noventa con el objetivo de ayudar a comprender mejor las vivencias de las personas con discapacidad intelectual y como un medio para poder ampliar el espectro de sus experiencias vitales (O’Brien y Lovett., 1992; O’Brien, 1987; O’Brien et al., 1997). Así, se configura como una metodología que pone a la persona, con sus deseos, objetivos y metas en el centro del sistema de apoyos. Desde una perspectiva ética, la PCP pone de manifiesto el derecho de cada persona a decidir sobre su propia vida, e implícitamente convierte la comunicación en la piedra angular de la autodeterminación, ya que es el medio a través del cual se puede influir sobre el entorno, hacerlo sensible a las propias demandas y receptivo a la propuesta de valor como individuo (Dressler et al., 2016).

Holburn et al. (2006), en su libro Person-Centered Planning Made Easy. The picture method proponen una versión de la PCP que incorpora dos elementos diferenciales. Por un lado, reconoce la importancia de las estructuras profesionales a la hora de diseñar y proveer apoyos, incidiendo en la necesidad de que dichos apoyos se generen dentro de un entorno comunitario normalizado. Y segundo, propone un sistema de seguimiento en el que se recoge la evolución de la organización, del plan individual y de los resultados en calidad de vida a través de la observación, cuestionarios y ejercicios. Este seguimiento potencia la orientación de la organización hacia una planificación individual que va más allá de la evaluación y que se integra en la intervención misma acabando por convertirse en una parte importante de ésta.

Según los mismos autores la educación es una de las actividades más importantes del método picture. Sin embargo, la encuadran como un proceso de capacitación que ha de producirse en un entorno normalizado, en el contexto social en el que se desenvuelve la persona. Así, por ejemplo, si la persona con discapacidad intelectual anhela tener más relaciones de amistad, quizás sea necesario ayudarla a tener conversaciones más fluidas y ricas y a desarrollar ciertas habilidades sociales útiles para tal fin. Este entrenamiento no debe producirse en un contexto aislado del entorno de la persona, sino con la participación de dicho entorno (Pérez y De lima, 2017).

En efecto, es necesario saber que a la hora de dinamizar o coordinar un plan centrado en la persona, emerge la figura del facilitador, quien debe responsabilizarse de mantener al círculo de apoyo cohesionado en torno a las metas y objetivos de la persona con discapacidad intelectual, además de evaluar las habilidades de comunicación y los desafíos del individuo (American Speech-Language-Hearing Association, 2016), y para ello deberá contar con ciertas competencias como: la capacidad de escuchar, inspirar y empoderar a todos los miembros del círculo de apoyo, y la flexibilidad y la positividad a la hora de integrar las aportaciones de todos y todas.

El círculo de apoyo está constituido por la propia persona con discapacidad (persona central) y por personas próximas a ésta, que son las encargadas de ayudar a las personas con necesidades específicas de comunicación a definir su plan y apoyarla en el despliegue de las estrategias necesarias para alcanzarlo (Griffiths, et al., 2019). Múltiples investigadores han discutido la importancia de involucrar a las familias en el proceso, respetando sus valores, visiones, creencias y prioridades (Calculator, 2014). 


\section{Integrando SAAC y PCP}

Según Lund et al. (2017), la toma de decisiones sobre el uso de un SAAC debe realizarse considerando tres aspectos fundamentales: la evaluación centrada en la persona, la valoración de las destrezas de los interlocutores, y el análisis de las características del dispositivo.

En este sentido, la combinación de los SAAC y la PCP permite desarrollar sistemas de comunicación eficaces y personalizados, involucrando al entorno de la persona y haciéndole partícipe del proceso de aprendizaje y utilización del SAAC que más se adecua a las capacidades concretas y a la realidad en la que dicha persona se desenvuelve (Dressler et al., 2016).

Una aproximación sistémica al proceso de comunicación evidencia que éste es eficaz cuando se establecen relaciones de interdependencia entre los participantes, es decir, cuando todos los intervinientes obtienen beneficios de dicha interacción. En este sentido Mandak y Light (2018) afirman que "los beneficios de esto incluyen una mayor generalización y mantenimiento de habilidades, el logro de objetivos de comunicación y desarrollo de relaciones de confianza" (pp.6). Básicamente se habla de motivaciones o necesidades comunes y de reciprocidad en el intercambio de información (Basil, 1998).

En esta medida un SAAC tendrá éxito cuando quede completamente integrado en el día a día, si se diseña teniendo en consideración el ecosistema relacional en el que ha de funcionar (McNaughton, et al., 2019), además de preservar la personalización en la creación del mismo (Brady et al., 2016; Clarke et al., 2016).

\section{Programa de comunicación}

Este programa de comunicación se plantea con una duración de dos años prolongables en el tiempo y, con la finalidad de abordar las necesidades específicas de comunicación de personas con discapacidad intelectual y del desarrollo con dificultades de comunicación.

El objetivo general del programa es contribuir a una mejor calidad de vida de las personas participantes (personas con discapacidad intelectual y del desarrollo y con dificultades de comunicación) a través de la mejora de la comunicación para que, principalmente, las personas de su entorno puedan relacionarse y entenderse con ellas. Para ello, se proponen $\mathbf{7}$ objetivos específicos:

- Lograr un mayor desarrollo personal.

- Fortalecer la capacidad de la toma de decisiones de las personas usuarias.

- Mejorar las relaciones de las personas usuarias con otras personas gracias a la comunicación.

- Aumentar la participación de las personas usuarias en actividades o en espacios sociales. 
- Mejorar el trato de la gente, así como el respeto a las opiniones de las personas usuarias.

- Contribuir al bienestar emocional y físico de las personas usuarias.

- Aumentar la autoestima y satisfacción de las personas usuarias.

\subsection{Participantes en el programa de comunicación}

En el programa de comunicación han participado las personas usuarias o participantes directas, las personas facilitadoras encargadas de dinamizar y coordinar el plan desarrollado para cada una de las personas usuarias y las personas que pertenecen al círculo de apoyo de las personas usuarias.

Concretamente, han participado 14 personas usuarias con discapacidad intelectual, quienes han sido evaluadas para valorar los resultados obtenidos y el impacto en la mejora de su calidad de vida. Atendiendo al perfil de las personas usuarias participantes, cabe decir que el 53,8\% son mujeres y el 46,8 \% hombres y la edad media es de 45,62 años. Mayoritariamente (76,9\%) conviven en entornos residenciales y todas ellas tienen un grado de discapacidad superior al $75 \%$, siendo el grado medio de 83,15\%. En cuanto a las necesidades comunicativas, en el 69,2\% de los casos podrían calificarse como "moderadas" y en el 23,1\% como "extensas". La intención comunicativa puede definirse como "alta" en la mayor parte de los casos $(84,6 \%)$.

\subsection{Procedimiento y desarrollo del programa de comunicación: método}

Este trabajo ha abordado las necesidades específicas de comunicación de personas con discapacidad intelectual y del desarrollo con dificultades de comunicación, mediante un proceso secuenciado (figura 1) en el que se ha construido y desplegado un plan de comunicación a partir de las necesidades y metas específicas de cada una de las personas participantes, involucrando a ésta y a su entorno, en sus rutinas y contextos diarios.

\section{Figura 1. Fases del proceso: itinerario}

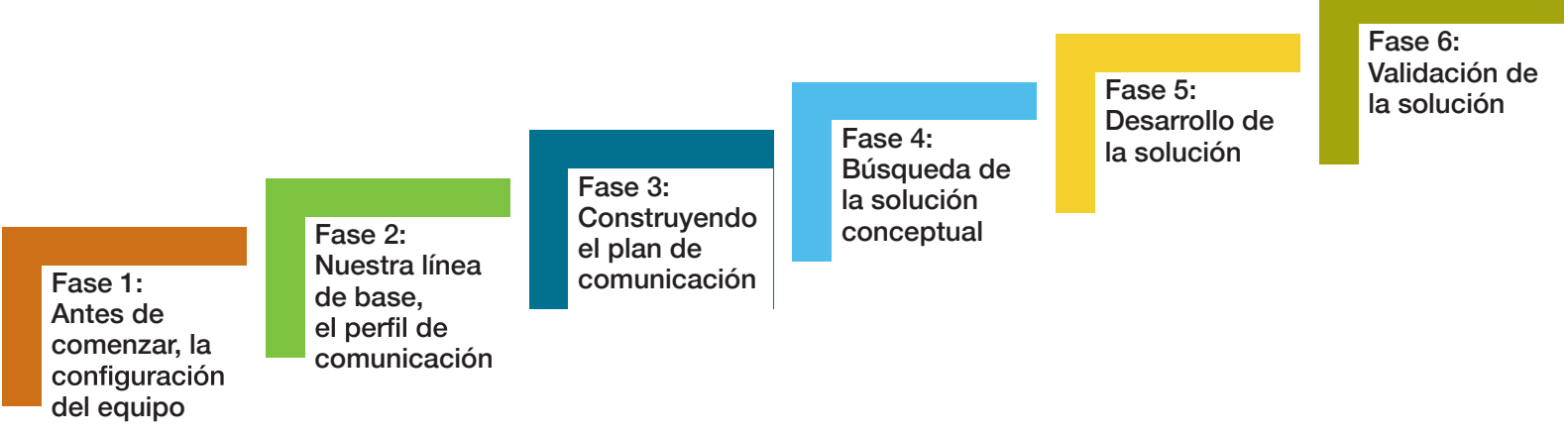

Fuente: elaboración propia. 
En la tabla 1 se muestra una síntesis de las fases del proceso abordándose en profundidad en el apartado siguiente.

\begin{tabular}{|c|c|c|c|}
\hline Fase & Principales actividades & Participantes & Herramientas a utilizar \\
\hline $\begin{array}{l}\text { 1.Configuración } \\
\text { del equipo }\end{array}$ & $\begin{array}{l}\text { La persona facilitadora presenta a la persona } \\
\text { con dificultades de comunicación y a su } \\
\text { familia el proyecto y se propone como } \\
\text { persona facilitadora. Junto con la persona va } \\
\text { configurando una propuesta de grupo de apoyo } \\
\text { que posteriormente validará con las personas } \\
\text { candidatas al mismo. }\end{array}$ & $\begin{array}{l}\text { - Persona facilitadora } \\
\text { - Persona central } \\
\text { - Familia }\end{array}$ & Mapa de relaciones \\
\hline 2.Perfiles & $\begin{array}{l}\text { La persona facilitadora y la persona central } \\
\text { elaboran el perfil de comunicación. }\end{array}$ & - Facilitador & Perfil de comunicación \\
\hline $\begin{array}{l}\text { 3.Plan de } \\
\text { Comunicación }\end{array}$ & \multicolumn{3}{|l|}{ Elaboración del Plan de comunicación } \\
\hline 3.1. Mapas & $\begin{array}{l}\text { La persona facilitadora prepara la sesión de } \\
\text { trabajo en la que, junto con la persona central, } \\
\text { trabaja el círculo de relaciones y el mapa de } \\
\text { entornos. }\end{array}$ & - Persona facilitadora & Mapas \\
\hline $\begin{array}{l}\text { 3.2. Calidad de } \\
\text { vida }\end{array}$ & $\begin{array}{l}\text { Análisis de las dimensiones de calidad de vida } \\
\text { desde el proyecto de comunicación. }\end{array}$ & - Persona facilitadora & $\begin{array}{l}\text { Modelo de calidad de vida } \\
\text { de ocho dimensiones de } \\
\text { Schalock y Verdugo (2003) }\end{array}$ \\
\hline 3.3. Imágenes & $\begin{array}{l}\text { Identificación de imágenes de futuro y de } \\
\text { estrategias. }\end{array}$ & $\begin{array}{l}\text { - Persona central } \\
\text { - Persona facilitadora } \\
\text { - Círculo de apoyo }\end{array}$ & $\begin{array}{l}\text { Todo lo trabajado } \\
\text { anteriormente } \\
\text { Plan de comunicación }\end{array}$ \\
\hline $\begin{array}{l}\text { 4.Solución } \\
\text { conceptual }\end{array}$ & $\begin{array}{l}\text { La coordinación TIC, la persona responsable } \\
\text { de fisioterapia y la persona facilitadora deberán } \\
\text { trabajar conjuntamente para la identificación del } \\
\text { soporte, la identificación de los accesorios y las } \\
\text { adaptaciones necesarias. }\end{array}$ & $\begin{array}{l}\text { - Persona facilitadora } \\
\text { - Coordinación TIC } \\
\text { - Fisioterapeuta }\end{array}$ & \\
\hline 5.Despliegue & $\begin{array}{l}\text { Capacitamos, practicamos, evaluamos y } \\
\text { actuamos con el objetivo de que el SAAC } \\
\text { acabe convirtiéndose en una herramienta de } \\
\text { uso cotidiano y habitual para la persona central } \\
\text { y su círculo de relaciones. La observación } \\
\text { participante es la clave para monitorizar la } \\
\text { evolución del proyecto. }\end{array}$ & - Grupo de apoyo & Diario de campo \\
\hline 6.Validación & $\begin{array}{l}\text { El proyecto se consolida cuando la solución se } \\
\text { utiliza de forma normalizada. }\end{array}$ & - Grupo de apoyo & \\
\hline
\end{tabular}

Fuente: elaboración propia. 
5.3. Fase 1. Antes de comenzar: la configuración del equipo de trabajo

\section{a. La motivación y el compromiso de la persona central y de su familia}

La propuesta de comenzar este proyecto de comunicación surge de personas que forman parte del círculo de relaciones de la persona participante, pero ha sido presentada y valorada por la propia persona y su entorno más próximo. Su motivación e interés junto con el de su red natural son factores sin los que ninguna propuesta resultaría eficaz.

\section{b. La elección del facilitador: su rol y competencias}

En nuestra experiencia, el proyecto suele motivar desde el comienzo, pero hemos de tener en cuenta que los objetivos ligados al plan de comunicación tardan en verse y requieren de constancia, y una alta dosis de paciencia e ilusión. Sostener esta motivación y orientar a las personas participantes en las diferentes fases del proyecto ha sido labor de la persona facilitadora. Esta es la persona responsable de dinamizar al grupo de apoyo en torno al proyecto, aprovechando la potencia del grupo para conseguir buenos resultados. Así que de la elección adecuada de la persona facilitadora depende en gran medida el éxito del proyecto.

Las principales competencias de la persona facilitadora son:

- Ser capaz de identificar los intereses de la persona central preservando un marco de confidencialidad y respeto a la intimidad.

- Liderar e implementar el proyecto orientando al grupo de apoyo a lo largo de todo el proceso.

- Implicar y motivar a todas las personas participantes orientando su actuación hacia los objetivos establecidos.

\section{c. ¿Cómo definimos el grupo de apoyo?}

En una sesión, la persona con dificultades de comunicación y la persona facilitadora han identificado a las personas que han constituido el grupo de apoyo.

Una vez identificadas las personas, se les ha explicado el proyecto y se les ha planteado su participación. La elección de participar o no en el grupo de apoyo ha sido voluntaria y ha conllevado una serie de compromisos tales como: respetar la intimidad y la confidencialidad de la información personal que se vaya a utilizar, participar regularmente y de manera proactiva en las reuniones, tratar a la persona central desde una relación horizontal y procurar el mayor beneficio para ella en cada actuación nuestra. En la tabla 2 se describen los cometidos de los integrantes del equipo de trabajo. 


\begin{tabular}{|c|c|}
\hline AGENTE & ACTIVIDAD PRINCIPAL \\
\hline Persona facilitadora & $\begin{array}{l}\text { Es responsable de guiar y motivar al resto de participantes en las diferentes fases del proceso } \\
\text { pero, sobre todo, es una persona que cree en las posibilidades y oportunidades de la persona con } \\
\text { dificultades de comunicación, acompañándola de una manera muy cercana para conseguir las } \\
\text { metas pretendidas. }\end{array}$ \\
\hline Persona central & $\begin{array}{l}\text { Es la persona con dificultades de comunicación y aporta toda la información sobre sí misma, } \\
\text { sus experiencias y las actividades más gratificantes para poder desplegar mediante el plan de } \\
\text { comunicación. }\end{array}$ \\
\hline $\begin{array}{l}\text { Integrantes del grupo } \\
\text { de apoyo }\end{array}$ & $\begin{array}{l}\text { Son personas próximas a la persona central, identificadas por ella misma y la persona facilitadora, } \\
\text { y se encargan de ayudar a la persona central a definir su plan y ayudarle a desplegar las } \\
\text { estrategias necesarias para alcanzarlo. }\end{array}$ \\
\hline Coordinación TIC & $\begin{array}{l}\text { Profesional con experiencia en las diferentes propuestas en productos de apoyo a la } \\
\text { comunicación, tanto en su identificación como en su posterior implantación. }\end{array}$ \\
\hline Fisioterapeuta & $\begin{array}{l}\text { Responsable experta en las adaptaciones necesarias en personas con dificultades motoras de } \\
\text { acceso a las interfaces de los dispositivos de comunicación. }\end{array}$ \\
\hline
\end{tabular}

Fuente: elaboración propia.

\subsection{Fase 2. Nuestra línea base: el perfil de comunicación}

El perfil nos ha proporcionado información relevante sobre cuatro cuestiones:(1) cómo se comunica la persona, (2) cuál es su capacidad de atribución de significados, (3) cuáles son sus preferencias, (4) qué estrategias y recursos utiliza. Para recabar esta información, el perfil se estructura en siete apartados: (1) descripción, (2) autodirección, (3) conducta comunicativa - diagnóstico funcional, (4) competencias sociales, (5) competencias lingüísticas, (6) competencias operativas, (7) competencias estratégicas.

\subsection{Fase 3. Construyendo el plan de comunicación}

El plan de comunicación sirve para identificar los objetivos y expectativas de la persona participante en torno a la comunicación. Esto es: ¿para qué quiere mejorar su comunicación?, ¿qué valor añadido quiere que le aporte esto a su vida? Se han identificado objetivos deseados y se han definido estrategias para ayudar a perseguir esas metas. No se ha tratado de abarcar grandes metas sino de identificar los pequeñísimos cambios que pueden hacer que la vida de la persona sea más gratificante para ella.

¿Qué pasos hemos de dar en esta fase del proyecto?

a. Mapas visuales.

b. Análisis de las dimensiones de calidad de vida.

c. Elección de las imágenes de futuro y definición de las estrategias. 


\section{a. Mapas visuales}

Se han trabajado con dos tipos de mapas: el mapa de relaciones o círculo de relaciones y el mapa de entornos.

\section{El círculo de relaciones}

Se refleja la red social de la persona participante. Es decir, la herramienta es una representación visual de las personas que forman parte de la vida de la persona con dificultades de comunicación.

\section{El mapa de entornos}

De la misma manera nos ha permitido identificar los entornos que están presentes en la vida de la persona como los inexistentes.

\section{b. Dimensiones de calidad de vida}

El objetivo de este paso es identificar qué aspectos de la calidad de vida de la persona condicionan o pueden verse condicionados por el proyecto de comunicación.

A la hora de indagar, hemos hecho especial énfasis en las siguientes consideraciones, que presentaremos a continuación siguiendo el modelo de ocho dimensiones propuesto por Schalock y Verdugo (2003):

Desarrollo personal

Esta dimensión es importante dado que estamos proponiéndole a la persona con dificultades de comunicación aprender cosas nuevas como, por ejemplo, utilizar un SAAC, sea este un móvil, una tablet o un tablero.

\section{Autodeterminación}

Hace referencia a situaciones en las que se requiera de una toma de decisiones. Elegir, por ejemplo, con quién salgo y paso mi tiempo libre, con quién comparto mis momentos, dónde desarrollo mi ocupación, qué cosas quiero hacer en mi vida, etc.

\section{Relaciones interpersonales}

Se ha valorado en qué medida la persona cuenta con las relaciones que desea tener, si éstas son satisfactorias y si echa en falta una red más amplia de gente con la que poder relacionarse.

\section{Inclusión social}

Gracias a las TIC se ha podido ofrecer a la persona un SAAC que integre apoyos para desenvolverse más fácilmente en lugares como su barrio, pueblo o ciudad. Por ejemplo, restaurantes y bares, centros comerciales, cines, discotecas, parques, tiendas, etc.

\section{Derechos}

El objetivo ha sido que la persona participante sea tratada como el resto de la gente, teniendo opiniones y deseos propios (por ejemplo, puedo opinar sobre lo que quiero comer, dónde quiero ir, cómo quiero divertirme, más allá de una respuesta "sí o no" en todas las conversaciones). 
Bienestar emocional

El sistema de comunicación ha generado una sensación de mayor control del entorno, mayor seguridad, con más recursos para hacer frente a situaciones ante las que, de otro modo, pudiera verse indefensa y, por tanto, responder a ellas de forma desajustada.

\section{Bienestar físico}

En caso de enfermedad o malestar, poder transmitir síntomas de manera más específica y demandar medidas para prevenirlos o tratarlos.

Bienestar material

Poder hacerse cargo de las compras personales de forma más autónoma.

\section{c. Imágenes de futuro y estrategias}

El plan constó de dos elementos que lo han articulado: las imágenes de futuro y las estrategias. Las imágenes de futuro se construyen identificando el gap existente entre lo que la persona vive en el presente y sus expectativas. Y las estrategias serían los medios que el grupo de apoyo identifica para alcanzar los objetivos.

Se han puesto en valor los pequeños cambios que surgen en la vida cotidiana de las personas, que pueden ser claves para un sentimiento de vida mejor y de trabajo conseguido por el grupo.

\subsection{Fase 4. Búsqueda de la solución conceptual}

En esta fase se han definido cuáles van a ser los recursos que se han proporcionado a la persona participante para tratar de alcanzar los objetivos que nos hemos marcado.

Para ello hay dos perfiles técnicos necesarios y con una importancia determinante.

El primero es la coordinación TIC, profesional con experiencia en tecnologías de apoyo a la comunicación. Es quien nos ha ayudado tanto en su identificación como en su posterior implantación.

El otro perfil técnico será el de fisioterapeuta. Su labor ha consistido en estudiar las adaptaciones que pueden requerir las personas con dificultades motoras para interactuar con los dispositivos de comunicación.

Su atención se ha centrado principalmente en tres aspectos:

- La identificación del soporte más adecuado para la persona central, lo que requiere conocer las características de recursos tableros, plantillas, plafones, cuadernos de comunicación, tablets, o smartphones y el software específico de comunicación.

- La identificación de los accesorios ha sido tan importante como la de los soportes. Cuál es el mejor medio para transportar el soporte depende mucho de las características de la persona y de sus imágenes de 
futuro. No es lo mismo para una persona con un buen control de su motricidad, en cuyo caso una bandolera puede ser lo más cómodo, que una persona con movilidad reducida en cuyo caso puede que se haya necesitado un accesorio para sujetar el dispositivo a la silla de ruedas, por ejemplo. Igualmente, si la persona necesita el soporte verbal del dispositivo, necesita altavoces para determinados ambientes.

Finalmente, se han valorado las adaptaciones en los casos en los que la persona no puede acceder directamente a las interfaces de los dispositivos: brazos extensibles, punteros, pulsadores, etc.

Este trabajo es vital porque de él depende la usabilidad de la solución que hemos puesto en marcha.

\subsection{Fase 5. Desarrollo de la solución}

En esta fase se comenzó a trabajar en entornos reales con la solución conceptual previamente diseñada. Para hacerlo, hemos utilizado una sistemática de menos a más. Esto es, siempre en situaciones reales, primero hemos probado la solución en contextos muy concretos y durante breves lapsos de tiempo. A medida que la persona ha ido adquiriendo soltura, se ha generalizado el uso de la solución a un mayor número de contextos vitales.

\subsection{Fase 6. Validación de la solución}

La fase anterior dura el tiempo necesario hasta que el equipo de apoyo, persona con dificultades de comunicación incluida, valora que la solución se ha convertido en un apoyo habitual en la vida de la persona, que tanto la persona central como su entorno sabe utilizarlo y lo utiliza, y el equipo de apoyo es capaz de generar el soporte necesario para que el SAAC siga ofreciendo un rendimiento óptimo (actualizaciones de software, pictogramas nuevos que la persona pudiera necesitar...).

\section{La evaluación de los resultados del programa de comunicación}

\section{- Enfoque y metodología de evaluación}

Existen varios enfoques y metodologías para la evaluación de este tipo de programas o iniciativas de carácter social. El estudio del impacto de los programas sociales es un tema relativamente nuevo. En un primer momento, los programas sociales se describían a través de los objetivos que perseguían y de las principales variables asociadas como el presupuesto y el número de personas beneficiarias y, como máximo, a través de la identificación de beneficios esperados. Sin embargo, en los últimos años este tema ha cobrado una mayor atención e interés por parte de profesionales y personal académico, lo que ha contribuido a avanzar y desarrollar el marco teórico y metodológico. Dentro de esta corriente, ha tenido una especial relevancia el concepto de calidad de vida como eje central dentro de la evaluación de los beneficios sobre las personas beneficiarias de los programas, ya que la mejora de la calidad de vida puede considerarse un objetivo 
estratégico habitual de los programas sociales, siendo éste el objetivo del programa de comunicación que nos compete.

Siendo así, esta evaluación ha tenido el objetivo de responder al interés por entender y valorar el efecto que ha tenido el programa de comunicación sobre los diferentes ámbitos que componen el bienestar de las personas usuarias. Teniendo en cuenta los objetivos que pretende el programa de comunicación, para esta evaluación se ha considerado el modelo de las ocho dimensiones de calidad de vida de Schalock y Verdugo (2003).

Para el análisis de resultados se ha optado por combinar la metodología cuantitativa y cualitativa como método para analizar los pequeñísimos cambios alcanzados en las personas usuarias en relación con cada una de las ocho dimensiones de calidad de vida (desarrollo personal; autodeterminación; relaciones interpersonales; inclusión social; derechos; bienestar físico; bienestar material; bienestar emocional) del modelo multidimensional desarrollado por Schalock y Verdugo (2003). Respecto a los instrumentos utilizados, por un lado, se ha aplicado un cuestionario dirigido a las personas usuarias, a las personas facilitadoras y los círculos de apoyo. Con él se han recogido datos de percepción sobre los logros del programa y relevancia de los cambios experimentados desde ópticas distintas. Por otro lado, se ha llevado a cabo un grupo focal participado por personas facilitadoras del programa y una entrevista en profundidad con la persona responsable del programa.

Para el análisis de los datos cuantitativos se ha utilizado el programa estadístico SPSS que ha permitido llevar a cabo una comparación de frecuencias y parámetros descriptivos básicos.

\section{- Los objetivos de la evaluación}

Dos han sido los objetivos fundamentales de la evaluación, por un lado, analizar la eficacia del programa, es decir los logros alcanzados y por otro lado, valorar el impacto inmediato en las personas usuarias.

\section{- Participantes en el proceso de evaluación para valorar los resultados alcanzados con el programa}

En la evaluación de los resultados del programa de comunicación han participado un total de 27 personas: 12 personas usuarias (48,1\% del total); 13 personas facilitadoras encargadas de dinamizar y coordinar el plan desarrollado para cada una de las personas usuarias (44,4\%); y 2 personas que pertenecen al círculo de apoyo de las personas usuarias $(7,4 \%)$. Cabe señalar que la mayor parte de las personas que componen el grupo de facilitadoras y círculo de apoyo son mujeres $(71,4 \%)$ y la edad media es de 37,8 años, siendo lo más habitual que tengan entre 18 y 35 años (50\%) o bien entre 36 y 50 años (35,7\%).

\section{- Análisis de resultados y discusión}

El objetivo principal del programa de comunicación es contribuir a una mejor calidad de vida de las personas participantes, a través de la mejora de la comunicación para que, principalmente, las personas de su 
entorno puedan relacionarse y entenderse con ellas. Se trata de utilizar todo el potencial que ofrecen los dispositivos y las nuevas tecnologías como herramientas para mejorar y enriquecer la relación con su entorno.

En base a una escala del 1 al 5 las personas usuarias valoran el logro de objetivos del programa con una media de 4,46 puntos, siendo 5 máximo nivel de satisfacción con el cumplimiento. Los resultados indican que su percepción sobre los logros es aún mejor que la de las personas facilitadoras y del círculo de apoyo que valoran los logros con 3,36 puntos de media.

Los hombres parecen mostrarse más optimistas en cuanto a la consecución de los objetivos planteados por el programa (tanto entre personas usuarias como entre el resto de los participantes); y en función de la edad, las personas usuarias de entre 36 y 50 años son las que mejor valoran este aspecto, mientras que en el caso de personas facilitadoras y círculo de apoyo este grupo es precisamente el más crítico con esta cuestión.

Un primer análisis de los datos que muestra la gráfica 1 permite observar que las personas usuarias perciben que el programa ha tenido un mayor impacto en las dimensiones de Bienestar Emocional $(4,46)$, Derechos $(4,23)$, Desarrollo Personal (4) y Bienestar Material (4), seguido a cierta distancia de las mejoras en Relaciones Interpersonales $(3,85)$ y Autodeterminación $(3,69)$, mientras que en las dimensiones de Inclusión Social $(3,31)$ y Bienestar Físico $(1,31)$ son en las que menores avances han percibido.

Por otro lado, las personas facilitadoras y del círculo de apoyo destacan sobre todo los logros en términos de Bienestar Emocional $(4,36)$, Relaciones Interpersonales $(4,21)$ y Bienestar Material $(4,07)$, seguido de los avances en las dimensiones Desarrollo Personal $(3,86)$ y Autodeterminación $(3,36)$. Este grupo considera que el impacto del programa en las dimensiones Inclusión Social $(2,86)$, Derechos $(2,36)$ y Bienestar Físico $(1,5)$ es más tímido.

La coincidencia entre estas dos ópticas es clara en relación con los beneficios sobre las dimensiones Bienestar Emocional y Bienestar Material y, aunque con ligeras diferencias, también puede decirse que hay acuerdo al evaluar los logros en Desarrollo Personal y Relaciones Interpersonales.

Llama la atención la diferencia en la valoración que estos dos grupos hacen en relación con la dimensión relativa a los Derechos. Así, mientras que las personas usuarias perciben que el programa tiene un impacto especialmente positivo en esta dimensión $(4,23)$, para las personas facilitadoras y el resto de las personas que componen el círculo de apoyo la repercusión del programa en este aspecto es considerablemente más reducida $(2,86)$. 


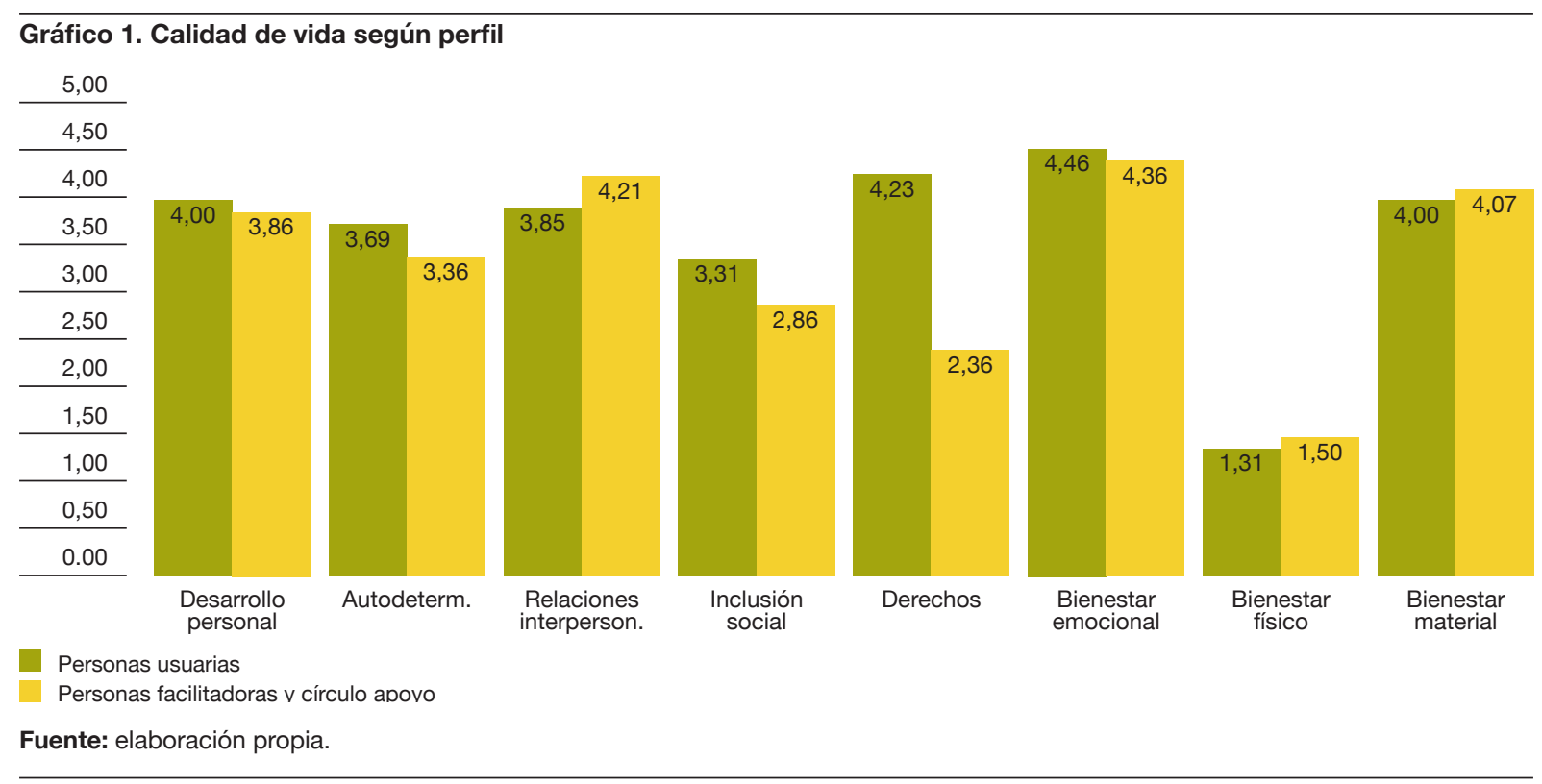

A continuación, se analizan con más detalle los cambios y mejoras percibidas en relación con cada una de estas dimensiones:

\section{- Desarrollo personal (aprendizaje de nuevas habilidades)}

Las personas usuarias ofrecen una valoración en esta dimensión algo superior a la del resto de personas consultadas (4 puntos de media frente a 3,86).

Aquellas personas con necesidades comunicativas definidas como "extensas" ofrecen una valoración particularmente alta en este aspecto $(4,67)$.

Las personas facilitadoras y pertenecientes al círculo de apoyo valoran de forma especialmente positiva el aprendizaje adquirido y continuo en cuanto a la utilización, comprensión y manejo de los dispositivos elegidos tanto para las personas usuarias como para su entorno. Concretamente, señalan el aprendizaje sobre cómo abordar y utilizar una pantalla táctil.

\section{- Autodeterminación (toma de decisiones)}

En esta dimensión las personas usuarias también ofrecen una puntuación algo superior a la de las personas facilitadoras y las que componen el círculo de apoyo (3,69 y 3,36 respectivamente).

Las personas usuarias entre 36 y 50 años $(4,20)$ y aquellas con necesidades comunicativas "extensas" $(4,33)$ son quienes mejor valoran los logros alcanzados por el programa en esta dimensión. 
Las personas facilitadoras consideraron la importancia de facilitar algunas opciones en los dispositivos para que las propias personas usuarias pudieran elegir lo que deseaban y lo que no, valorando sus gustos y las diferentes opciones en cada caso. En este sentido, se facilita que las personas usuarias elijan lo que desean en cada momento, de forma que las decisiones no son tan guiadas y tienen más posibilidades de elegir. Ejemplo descrito por una persona facilitadora:

"Cuando la persona usuaria necesita una cita médica, ahora dispone de capacidad y tiene la posibilidad de ver los días y las horas disponibles a través de la página de Osakidetza y puede elegir la que desee... Tiene la necesidad de comprar una cama y no puede desplazarse al establecimiento, pero a través de la página Web, ella puede ver las posibilidades que hay y puede elegir...".

\section{- Relaciones interpersonales (mejora de las relaciones con otras personas)}

Las personas facilitadoras y las que componen el círculo de apoyo valoran con una puntuación de 4,21 el impacto del programa en las Relaciones Interpersonales de las personas usuarias.

Esta valoración es superior a la de las propias personas usuarias $(3,85)$. Cabe destacar la valoración positiva en relación con el impacto del programa en esta dimensión de las mujeres usuarias $(4,29)$ así como las personas más jóvenes - entre 18 y 35 años - $(4,33)$.

La relación con personas que presentan dificultades comunicativas importantes requiere de realizar un esfuerzo importante de comprensión y paciencia, lo cual supone un obstáculo especialmente difícil de salvar en la relación entre compañeras y compañeros. El programa contribuye a que las personas usuarias amplíen su círculo de relaciones, enriqueciéndolas y favoreciendo relaciones de mayor calidad. Las personas usuarias se sienten más valoradas por sus compañeros y compañeras, perciben que les tienen más en cuenta y se relacionan más con ellos y ellas.

Muchas de las personas consultadas señalan la tablet como uno de los dispositivos más importantes a la hora de abrir las posibilidades de comunicación con otras personas de forma rápida y precisa. Muchas personas que anteriormente no se habían planteado la comunicación con personas compañeras, a raíz de disponer de una tablet se relacionan y mantienen conversaciones constantes. Comentarios de una persona facilitadora:

“... manda y recibe emails y comparte fotografías con la familia y compañeros exponiendo y contando su día a día, cómo se siente, qué ha hecho, qué necesita, sus gustos, etc.... comparte momentos lúdicos con compañeros y compañeras, ya que éstos y éstas se acercan y ello genera que la persona usuaria se sienta protagonista y bien..."

Inclusión social (participación en la sociedad, espacios nuevos, conocer nuevas personas...).

Las personas usuarias valoran el impacto en esta dimensión con 3,31 puntos sobre 5, una puntuación significativamente por debajo de la de otras dimensiones. Las personas facilitadoras y del círculo de apoyo coinciden en una puntuación moderada $(2,86)$. 
Los hombres - tanto usuarios como facilitadores - muestran una valoración más optimista en este aspecto. Además, las personas usuarias con necesidades "extensas" ofrecen también una puntuación algo superior $(3,67)$.

Algunos de los logros en esta dimensión se relacionan con poder valerse por sí mismas, ya que disponen de una mayor autonomía. Por ejemplo, cuando salen a una cafetería ya no tiene que pedir nadie por ellas. Algunas personas han tenido la posibilidad de participar en jornadas como ponentes. Según la persona facilitadora:

\footnotetext{
"Una persona usuaria con grandes dificultades comunicativas y una gran discapacidad intelectual ha tenido la posibilidad de sentirse integrada y el hecho de poder comunicarse le ha abierto posibilidades que le hacen sentirse bien. En este caso, tenía su presentación de quién era, dónde vivía, qué hace, etc. en un Power Point y, en el mes de junio, surgió la posibilidad de ir con su tablet y con una monitora de la residencia a un colegio público, concretamente al aula de 5 años a contarles a los/as niños/as dónde vivía, qué hacía durante el día...en fin, a contar su vida. Se apoyó en la tablet para contarles a los niños y las niñas lo que hacía, contó que necesitaba ayuda para ducharse y lo enseñó a través de una foto de la camilla que utiliza... Después los niños y las niñas han visitado la residencia y, se ha creado un vínculo increíble. Esta acción antes no hubiera podido hacerla y, ha aumentado la autoestima y se siente muy bien..."
}

- Derechos (Sentimiento de ser una persona valorada y tratada como el resto de la gente. Posibilidad de tener opiniones propias).

La comunicación es un derecho a través del cual es posible conseguir todos los demás derechos. El hecho de que las personas usuarias se sientan escuchadas y tratadas de igual a igual contribuye positivamente también en términos de autoestima.

Si bien las personas usuarias valoran la repercusión en la dimensión relativa a Derechos con 4,23 puntos sobre 5, las personas facilitadoras y del círculo de apoyo perciben un impacto considerablemente más modesto $(2,36)$.

Hay que destacar la valoración particularmente buena que hacen los hombres usuarios $(4,67)$ así como las personas usuarias de menos de 50 años.

- Bienestar emocional (sentimiento de seguridad, tranquilidad, relajo).

La valoración que hacen tanto las personas usuarias $(4,46)$ como las personas facilitadoras y del círculo de apoyo $(4,36)$ con respecto al impacto del programa en esta dimensión es muy similar y positiva.

En el caso de las personas usuarias cabe destacar sobre todo la buena valoración que hacen los hombres $(4,83)$ así como las personas con edades superiores a los 36 años. 
La mejora del Bienestar Emocional guarda relación con el impacto del programa en otras dimensiones tales como Relaciones Interpersonales, Derechos, Autodeterminación, ...etc. Quienes han participado se sienten más seguras, están orgullosas de sí mismas, se encuentran más integradas y contentas, en la medida en que ahora establecen relaciones interpersonales más satisfactorias, saben que el resto de las personas les están entendiendo y pueden participar en el día a día del centro junto con el resto de sus compañeras y compañeros.

A menudo, las personas usuarias utilizan las tablet para sacar fotografías y vídeos de todo lo que hacen día y día (en el centro o fuera del mismo) y las utilizan como soporte para contar a compañeros/as y familiares lo que hacen y cómo se sienten. La tablet es un complemento en la comunicación de las personas usuarias y a través de las fotografía y vídeos que sacan, cuentan y desarrollan la historia de su vida, lo que les hace sentir importantes, escuchadas y satisfechas consigo mismas.

Entre los logros más significativos también cabe mencionar el descenso de los niveles de frustración de personas que antes del programa intentaban comunicarse y explicarse sin éxito. Además, los dispositivos empleados en el programa poseen comunicadores específicos que permitan a la persona usuaria comunicar sus estados emocionales (en relación con su salud, sus gustos, su apariencia, sus sentimientos... etc.) de forma que permite a las personas usuarias comunicar cómo se sienten en cada momento, cómo desean vestirse, etc.

- Bienestar físico (sensación de buena salud, de encontrarse físicamente bien).

Los resultados en la dimensión relativa a Bienestar Físico son los menos relevantes, tanto desde el punto de vista de las personas usuarias como de las facilitadoras y personas del círculo de apoyo $(1,31$ y 1,50 respectivamente).

Independientemente de las características de estas personas, la puntuación media en esta dimensión no supera los 2 puntos sobre 5 .

El trabajo del programa en relación con esta dimensión se enfoca a no perjudicar el bienestar físico de las personas usuarias. Es decir, el hecho de utilizar dispositivos con personas con dificultades añadidas como escoliosis, etc. hace necesario un diagnóstico postural previo por parte de un o una fisioterapeuta para garantizar que los soportes utilizados no incidían negativamente en el bienestar físico (incluso en caso necesario se han diseñado soportes especiales adaptados).

- Bienestar material (contar con los recursos personales necesarios)

No se advierten apenas diferencias en la valoración de las personas usuarias y personas facilitadoras y del círculo de apoyo con respecto a la dimensión Bienestar Material (4,00 y 4,07 puntos respectivamente).

En el caso de las personas usuarias, cabría destacar que a medida que se incrementa la edad la valoración en este aspecto desciende progresivamente. 
El hecho de que las personas usuarias dispongan de un dispositivo de comunicación como es una tablet, cuadros de comunicación y pulsadores con mensajes grabados (y tengan cierta autonomía para utilizarlos) les ha conferido cierto prestigio social entre sus compañeros y compañeras.

\section{Discusión de los resultados: conclusiones}

El valor añadido que presenta esta metodología frente a otras radica en el trabajo individualizado, ya que el proceso a seguir con cada persona usuaria se diseña buscando que sea el más adecuado para ella, considerando sus necesidades particulares y su entorno concreto.

Los resultados de la evaluación destacan la satisfacción general de las personas usuarias con la consecución del logro de los objetivos planteados en el plan de comunicación. Los dos grupos participantes de la evaluación, personas facilitadoras y usuarias coinciden en destacar los beneficios en las dimensiones de calidad de vida de Bienestar Emocional, Relaciones Interpersonales y Bienestar Material.

El aumento percibido en la dimensión de bienestar emocional se vincula con en el hecho de que las personas han podido expresarse y hacerse entender en relación con aspectos básicos de su vida y en relación con sus sentimientos, reduciendo el estrés con el manejo de un dispositivo que podría producirse al sentirse incomprendido por su interlocutor y que provocaría el abandono de la conversación (Acuña-Partal et al., 2018). Las personas con dificultades de comunicación deben tener experiencias positivas con sus sistemas de comunicación, necesitan oportunidades de usar sus SAAC para responder a más que un sí y un no o un simple saludo (Chung y Douglas, 2014). La implicación comunicativa aumenta si se basa en intereses personales (Acuña-Partal et al., 2018).

El programa multiplica las posibilidades para mejorar cuantitativa y cualitativamente sus relaciones con compañeras y compañeros de centro, sus relaciones interpersonales. Las personas usuarias participan más activamente en la vida diaria del centro y hablan con compañeros y compañeras con los que nunca se habían relacionado dadas las barreras existentes. Esto supone un valor añadido frente a otro tipo de intervenciones que el casi la totalidad de las interacciones solo son con profesionales o familiares (Chung et al., 2012).

En relación con la percepción de la dimensión de Bienestar Material, el uso de la tablet y otros dispositivos propios como soporte para poder expresarse en su vida cotidiana, les hace sentir importantes y satisfechas consigo mismas. Los equipos de facilitadores tienen que integrar estrategias de autogestión y apoyar a las personas para ser responsables de sus propios equipos (Chung y Douglas, 2014). Este es un aspecto a destacar frente a metodologías en las que sus resultados muestran más de la mitad del tiempo observado sin acceso a su dispositivo por diversos motivos, lo que les hacía recurrir a su comunicación previa (Chung et al., 2012). 
Abbeduto, L. et al. (2001). "Theoretical perspectives on language and communication problems in mental retardation and developmental disabilities". Mental Retardation and Developmental Disabilities Research Reviews, $7(1)$, pp. $45-55$.

Acuña-Partal, C., et al. (2018). "Aptitudes y sinergias del intérprete para personas con Discapacidad Intelecual Severa con el apoyo de la herramienta de comunicación EC+". En E. Postigo et al. Disability and communication Scientific Analysis, Total Communication, ICT Tools and case studies (pp. 71-83). Madrid: McGraw-Hill

American Speech-Language-Hearing Association (ASHA). (2005). Evidence- based practice in communication disorders [Position Statement]. Recuperado de: https://www.asha.org/Research/EBP/Evidence-Based-Practice

American Speech-Language-Hearing Association. (2016). Scope of practice in speech-language pathology. Recuperado de https://www.asha.org/policy/SP2016-00343/.

Andzik N. et al. (2016) "Communication Opportunities for Elementary School Students who use Augmentative and Alternative Communication", Augmentative and Alternative Communication, 32(4), pp. 272-281

Barbosa, R. et al. (2018). "Augmentative and alternative communication in children with Down's syndrome: a systematic review". BMC Pediatrics, 18(1), pp. 1-16.

Basil, C. (1998). "Introducción". En C. Basil et al., Sistemas de signos y ayudas técnicas para la comunicación aumentativa y la escritura (pp. 1-5). Barcelona: Masson.

Batorowicz, B. et al. (2018). "Assessment of aided language comprehension and use in children and adolescents with severe speech and motor impairments", Augmentative and Alternative Communication, 34(1), pp. 54-67

Beukelman, D., y Mirenda, P. (2013). Augmentative and alternative communication: Supporting children and adults with complex communication needs. Baltimore, Maryland: Paul H. Brookes Publishing Co.

Brady, N.C. et al. (2016). "Communication services and supports for individuals with severe disabilities: guidance for assessment and intervention". American Journal on Intellectual and Developmental Disabilities, 121(2), pp.121-138.

Calculator, S. N. (2009). "Augmentative and alternative communication (AAC) and inclusive education for students with the most severe disabilities". International Journal of Inclusive Education, 13(1), pp. 93-113.

Calculator, S. N. (2014). "Parents perceptions of communication patterns and effectiveness of use Augmentative and Alternative communication systems by their children whit Angelman Syndrome". American Journal of Speech- Language Pathology, 23(4), pp. 562-573.

Clarke, M. et al. (2016). "Augmentative and alternative communication for children with cerebral palsy". Pediatrics and Child Health, 26(9), pp. 373-377.

Calleja, M. (2018). Sistemas de comunicación Aumentativa y Alternativa. De la investigación a la intervención logopédica. Malaga: Ediciones Aljibe.

Calleja, M., y Rodríguez, J. (2018). "La comunicación aumentativa y alternativa para hacer frente a las necesidades complejas de comunicación en usuarios de bajo perfil cognitivo". En E. Postigo et al., Disability and communication Scientific Analysis, Total Communication, ICT Tools and case studies (pp. 163-176). Madrid: McGraw-Hill 
Creer, S. et al. (2016). "Prevalence of people who could benefit from augmentative and alternative communication (AAC) in the UK: determining the need". International Journal of Language and Communication Disorders, 51(6), pp. 639-653.

Chung, Y. et al. (2012). "Social interactions of students with disabilities who use augmentative and alternative communication in inclusive classrooms". American Journal on Intellectual and Developmental Disabilities, 117(5), pp. 349-367.

Chung, Y. y Douglas, K. H. (2014). "Communicative competence inventory: A team approach to AAC use". Teaching Exceptional Children, 47(1), pp. 56-68.

Dressler, R. et al. (2016). "The Benefits of Alternative and Augmentative Communication: A Quality of Life Issue". Internet Journal of Allied Health Sciencesand Practice, 14(4), pp.1-6.

EDAD (2008). Encuesta de Discapacidad, Autonomía Personal y Situaciones de Dependencia. Instituto Nacional de Estadística. Recuperado de: https://www.ine.es/jaxi/Tabla.htm?path=/t15/p418/a2008/hogares/p01/modulo1/ I0/\&file $=01002 . p x \& L=0$

Enderby, P. (2013). "Introducing the therapy outcome measure for AAC services in the context of a review of other measures". Disability and Rehabilitation: Assistive Technology, 9(1), pp. 33-40.

Goldbart, J. et al. (2014). "Speech and language therapists' approaches to communication intervention with children and adults with profound and multiple learning disability". International Journal of Language \& Communication Disorders, 49(6), pp. 687-701.

Griffiths, C., y Smith, M. (2016). "Attuning: a communication process between people with severe and profound intelectual disability and their interaction partners". Journal of Applied Research in Intellectual Disabilities, 29(2), pp. 124-138.

Griffiths, T. et al. (2019). "Alternative and Augmentative Communication”. En L. Najafi y D. Cowan, Handbook of Electronic Assistive Technology, (pp. 181-213). Londres: Elsevier. doi:10.1016/b978-0-12-812487-1.00007-7

González, M. et al. (2014). "Sistemas de Comunicación No Verbales. Enriqueciendo los Lenguajes Aumentativos y Alternativos con propiedades de Accesibilidad y Usabilidad”. Revista de Informes Científicos y Técnicos de la Universidad Nacional de la Patagonia Austral, 6(2), pp. 30-56.

Holburn, S. et al. (2006). Person-Centered Planning Made Easy. The picture method. Baltimore: Paul H Brookers Publishing Co.

International Society for Augmentative and Alternative Communication (ISAAC) (2017). What is AAC? Recuperado de: https://www.isaac-online.org/english/what-is-aac/

Light, J. (1989). "Toward a definition of communicative competence for individuals using augmentative and alternative communication system". Augmentative and Alternative Communication, 5(2), pp. 137-144.

Light, J. et al. (1998). "Augmentative and alternative communication to support receptive and expressive communication for people with autism". Journal of Communication Disorders, 31(2), pp. 153-180.

Light, J.C. et. al. (2003). "Finding a place in the "social circle of life"”. En J.C., Light et al. (Eds.), Communicative competence for individuals who use AAC: From research to effective practice, (pp. 361-397). Baltimore: Brookes Pub. Co

Light, J., y McNaughton, D. (2014). "Communicative competence for individuals who require augmentative and alternative communication: A new definition for a new era of communication?". Augmentative and Alternative Communication, 30(1), pp. 1-18. 
Light, J., y McNaughton, D. (2015). "Designing AAC research and intervention to improve outcomes for individuals with complex communication needs". Augmentative and Alternative Communication, 31(2), pp. 85-96.

Lund, S. K., y Light, J. (2007). "Long-term outcomes for individuals who use augmentative and alternative communication: Part III- contributing factors”. Augmentative and Alternative Communication, 23(4), pp. 323-335.

Lund, SK., et al. (2017). "Assessment with children who need augmentative and alternative (AAC): Clinical decisions of AAC specialists". Language, Speech, and Hearing services in Schools, 48(1), pp. 56-68

Mandak, K., y Light, J. (2018). "Family-centered services for children with complex communication needs: the practices and beliefs of school-based speech-language Pathologists". Augmentative and Alternative Communication, 34(2), pp. 130-142.

McNaughton, D.et al. (2019). "Building capacity in AAC: A person-centred approach to supporting participation by people with complex communication needs". Augmentative and Alternative Communication, 35(1), pp. 56-68.

O'Brien, (1987). "A guide to life-style planning: Using the Activities Catalogue to integrate services and natural support system". En G.T. Bellamy y B. Wilcox (Eds.), A comprehensive guide to the Activities Catalogue: an alternative curriculum for youth and adults with severe disabilities (pp. 175-189). Baltimore: Paul H Brookers Publishing Co.

O’Brien, J. et al. (1997). "Person-centered planning has arrived or has it?" Mental Retardation, 36(6), pp. 480-484.

O’Brien, J. y Lovett, H. (1992). Finding a way toward everyday lives: The contribution of person-centered planning. Harrisburg: Office of Mental Retardation.

Perez, C. y De lima, R. (2017). "Linguagem e comunicação de pessoas com deficiencia intelectual e suas contribuições para a construção da autonomía". Inclusão social, 10(2), pp. 88-96.

Reichle, J. et al. (2016). "Playing the Long Game: Considering the Future of Augmentative and Alternative Communication Research and Service". In Seminars in Speech and Language, 37(4), pp. 259-273.

Rochelle, K. (2016). A comparison of two types of augmentative and alternative communication systems (ipad and pecs) for children with autism spectrum disorder: the benefits of integrating assistive technology into the ASD classroom (Tesis de grado). California State University, Fullerton.

Schalock, R. L. y Verdugo, M. A. (2003). Quality of life for human service practitioners. Washington, DC: American Association on Mental Retardation.

Simacek, J. et al. (2017). "Aided AAC for people with severe to profound and multiple disabilities: A systematic review of interventions and treatment intensity". Advances in Neurodevelopment Disorders, 2(1), pp. 100-115

Simeonsson, R. et al. (2012). "Communication, disability, and the ICF-CY". Augmentative and Alternative Communication, 28(1), pp. 3-10.

Snell, M. E. et al. (2006). "Teaching augmentative and alternative communication to students with severe disabilities: a review of intervention research 1997-2003". Research and Practice for Persons with Severe Disabilities, 31(3), pp. 203-214.

TASH (2016). TASH resolution on the right to communicate. Recuperado de: https://tash.org/about/resolutions/ tash-resolution-right-communicate

Soro-Camats, E. (1998). "El proceso de evaluar y tomar decisiones" En C. Basil et al., Sistemas de signos y ayuda técnicas para la comunicación aumentativa y la escritura (pp. 63-76). Barcelona: Masson. 
Soto, G. y Clarke, M. (2017). "Effects of a conversation-based intervention on the linguistic skills of children with motor speech disorders who use augmentative and alternative communication". Journal of Speech Language Hearing Research, 60(7), pp. 1-19. 